CERN-EP/2005-033

27 July 2005

\title{
ISOLDE BEAMS OF NEUTRON-RICH ZINC ISOTOPES: YIELDS, RELEASE, DECAY SPECTROSCOPY
}

\author{
U. Köster ${ }^{1)}$, T. Behrens ${ }^{2)}$, C. Clausen ${ }^{1) 3)}$, P. Delahaye ${ }^{1)}$, V.N. Fedoseyev ${ }^{1)}$, L.M. Fraile ${ }^{1)}$,

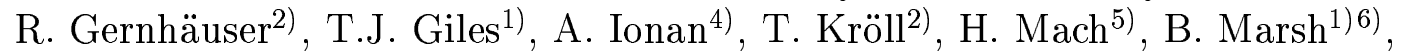 \\ M. Seliverstov ${ }^{4) 7)}$, T. Sieber ${ }^{1)}$, E. Siesling' ${ }^{1)}$, E. Tengborn ${ }^{1) 8)}$, F. Wenander ${ }^{1)}$ and \\ J. Van de Walle ${ }^{9)}$
}

\begin{abstract}
Intense radioactive ion beams of the neutron-rich zinc isotopes ${ }^{69-81} \mathrm{Zn}$ have been produced at the isotope separation on-line facility ISOLDE at CERN. The combined use of spallation-neutron induced fission of ${ }^{238} \mathrm{UC}_{x}$ targets and resonant laser ionization provided sufficient suppression of disturbing isobars (mainly gallium and rubidium) to perform decay spectroscopy up to ${ }^{81} \mathrm{Zn}$.
\end{abstract}

(Submitted to AIP Conference Proceedings, "Fission 2005"

$3^{\text {rd }}$ International Workshop on Nuclear Fission and Fission-Product Spectroscopy 11-14 May 2005, Château de Cadarache, Saint Paul les Duranze, France)

1) CERN, ISOLDE, 1211 Genève 23, Switzerland

2) Technische Universität München, Physik-Department, 85748 Garching, Bavaria

3) Aarhus Universitet, Institut for Fysik og Astronomi, 8000 Aarhus C, Denmark

4) Petersburg Nuclear Physics Institute, 188300 Gatchina, Russia

5) Uppsala University, Department of Radiation Sciences, 75121 Uppsala, Sweden

6) Nuclear Physics Group, University of Manchester, M13 9PL Manchester, UK

7) Johannes-Gutenberg Universität, Institut für Physik, 55128 Mainz, Germany

8) Chalmers University of Technology, Dept. of Experimental Physics, 41296 Göteborg, Sweden

9) K.U. Leuven, Instituut voor Kern- en Stralingsfysica, 3001 Heverlee, Belgium 
Various scientific communities demand radioactive ion beams for a multitude of applications. Depending on the position on the chart of nuclides different nuclear reactions may be used to produce the radioactive isotopes. Neutron-rich isotopes of medium mass are best produced by fission. ISOLDE at CERN provides ISOL (isotope separation online) beams of fission products since more than thirty years [1]. With up to $4 \mu \mathrm{A}$ of $1.4 \mathrm{GeV}$ protons onto a $50 \mathrm{~g} / \mathrm{cm}^{2}{ }^{238} \mathrm{UC}_{x}$ target slightly over $10^{13}$ fission products per second are produced. This is orders of magnitude higher than what other operating ISOL facilities can deliver today, and combined with efficient and selective ion sources it makes ISOLDE the world-leading radioactive ion beam facility.

For users the decisive quality parameters of radioactive ion beams are the intensity of the beam, its purity and emittance. It is well known that for ISOL beams the latter is excellent: at several ten $\mathrm{keV}$ beam energy the transverse emittance is typically of the order of $\varepsilon_{95 \%} \approx 10-20 \pi \mathrm{mm}$ mrad [2] while the energy spread normally does not exceed few $\mathrm{eV}$. The radioactive ion beam intensity scales obviously with the target thickness, the primary beam intensity and the production cross-section of the isotope in question. However, it is also strongly affected by the release and ionization efficiency, which just like the beam purity depends on the element and isotope in question and on the type of the employed target and ion source unit.

Therefore we discuss the characteristic yields and release parameters of ISOLDE beams in individual papers treating a given element or group of homologue or chemically similar elements. Previous reports discussing in detail the beams produced since 1992 at ISOLDE-PSB (using as driver accelerator a stack of four proton synchrotrons that provide $2 \mu$ s long pulses of ca. $3 \cdot 10^{13}$ protons every 1.2 seconds) cover the fission products Mn [3], Ni [4], Ga [5], Kr and Xe [6], Cd [7] and In [8]. In the present paper we will discuss beams of neutron-rich ${ }^{1)} \mathrm{Zn}$ isotopes.

\section{$2 \quad$ Production and separation}

For the production of beams of neutron-rich $\mathrm{Zn}$ isotopes $\mathrm{UC}_{x}$ /graphite $(x \approx 4)$ targets have been used in combination with different types of ion sources. Several measures can be used individually or combined to achieve satisfactory beam purity.

\subsection{Chemically selective transfer line}

The chemical elements differ strongly in their volatility. A high volatility translates at a given temperature into a higher vapor pressure and a shorter residence time on the surface. The group 12 elements $(\mathrm{Zn}, \mathrm{Cd}, \mathrm{Hg})$ are characterized by an atomic subshell closure $\mathrm{d}^{10} \mathrm{~s}^{2}$. This assures a clearly higher volatility compared to the neighboring elements. Hence, a transfer line (i.e. the tube connecting the target with the ion source) kept at a temperature just sufficient to allow the transport of short-lived $\mathrm{Zn}$ isotopes will retain neighboring isobars by condensing them on the surface of the tube. Thus, only the $\mathrm{Zn}$ isotopes are reaching the ion source while short-lived radioisotopes of less volatile elements are kept back in the transfer line sufficiently long to decay there. This chemical separation method is known as isothermal vacuum chromatography. In the case of zinc the lower $(\mathrm{Cu}, \mathrm{Ni}, \ldots)$ and higher isobars $(\mathrm{Ga}, \mathrm{Ge}, \mathrm{As}, \mathrm{Se})$ are retained completely. The remaining impurities are krypton, which is always transmitted, and bromine, which occasionally may be transmitted in form of volatile molecules. This is not very disturbing for decay

1) ISOLDE beams of neutron-deficient $Z n$ isotopes have been discussed in ref. [3]. 
spectroscopy experiments with very neutron-rich $\mathrm{Zn}$ isotopes (e.g. 80 to 82 ), since the respective krypton isotopes are stable or very long-lived ${ }^{2}$. Thus already at ISOLDE-SC relatively clean $\mathrm{Zn}$ beams have been produced using a plasma ion source combined with a temperature controlled transfer line and used for half-life measurements by beta-delayed neutron detection up to ${ }^{81} \mathrm{Zn}$ [9]. However, more recent applications of radioactive ion beams include direct precision mass measurements with a Penning trap and experiments with post-accelerated beams like Coulomb excitation. In these cases stable and radioactive isotopes represent equally disturbing background. Thus, another or additional measure has to be used to eliminate also krypton and bromine.

\subsection{Resonant laser ionization}

With the resonance ionization laser ion source (RILIS) [10], atoms of a given chemical element are selectively ionized by stepwise resonant excitation of a valence electron into the continuum. The excitation energy is provided by laser light tuned to strong atomic transitions in the respective element. For other elements the laser light is out of resonance and will therefore cause only negligible excitation and ionization. Thus the RILIS provides intrinsically extremely pure beams.

For the resonant laser ionization of zinc a formerly developed ionization scheme [11] has been used: frequency-tripled dye laser light at $213.86 \mathrm{~nm}$ excites from the atomic ground state $3 \mathrm{~d}^{10} 4 \mathrm{~s}^{2}{ }^{1} \mathrm{~S}_{0}$ to the $3 \mathrm{~d}^{10} 4 \mathrm{~s} 4 \mathrm{p}{ }^{1} \mathrm{P}_{1}^{o}$ state. The second transition is performed with dye laser light at $636.23 \mathrm{~nm}$ to the $3 \mathrm{~d}^{10} 4 \mathrm{~s} 4 \mathrm{~d}^{1} \mathrm{D}_{2}$ state from where the atoms are ionized non-resonantly to the continuum with the green copper vapor laser light at $511 \mathrm{~nm}$. The off-line measured ion source efficiency was $5 \%$.

Since presently only pulsed lasers can provide a sufficient peak power to saturate the atomic excitations, the atoms effusing from the target have to be "stored" for the time between two laser pulses to have at least one chance to interact with the laser light. Typically a long thin tube ("line" in ISOLDE jargon) is used to constrain the effusion of the atoms and serve as interaction region with the laser light.

To avoid "sticking" of the atoms to the surface of the tube and to ensure sufficient electron emission to provide conditions for ion repelling from the surface, the tube is typically made from a refractory metal and kept at temperatures of the order of $2000{ }^{\circ} \mathrm{C}$. One has to keep in mind that resonant laser ionization enhances the ionization efficiency of the element to which atomic transitions the lasers have been tuned, but it does not reduce the surface ionization of other elements in the hot cavity. Hence, surface ionized background of isobars with low ionization potential will be omnipresent. In the case of zinc this concerns particularly rubidium (4.18 eV ionization potential) which is efficiently surface ionized (>65\% efficiency) on any hot surface of a refractory metal $(\mathrm{Nb}, \mathrm{Ta}, \mathrm{W}, \ldots)$ and also gallium (6.00 eV ionization potential) which is ionized with an efficiency of the order of $1 \%$ in typical RILIS cavities at ca. $2000{ }^{\circ} \mathrm{C}$.

While the rubidium ionization efficiency is practically temperature independent, lowering the line temperature can reduce the gallium background. However, for too low line temperatures also the electron emission and, hence, the ion repelling and Zn ionization efficiency are compromised. Figure 1 shows the relative ${ }^{76} \mathrm{Zn}$ and ${ }^{76} \mathrm{Ga}$ intensities in dependence of the line temperature. Below $1950{ }^{\circ} \mathrm{C}$ the $\mathrm{Zn}$ ionization is compromised, but due to the faster drop of the Ga ionization efficiency the $\mathrm{Zn} / \mathrm{Ga}$ ratio can be improved by further reduction of the line temperature. For other isotopes the behavior is qualitatively

2) The background of krypton isomers can be strongly reduced by beta-gating. 
the same, only for the most short-lived ones the release efficiency is significantly affected at very low line temperatures.

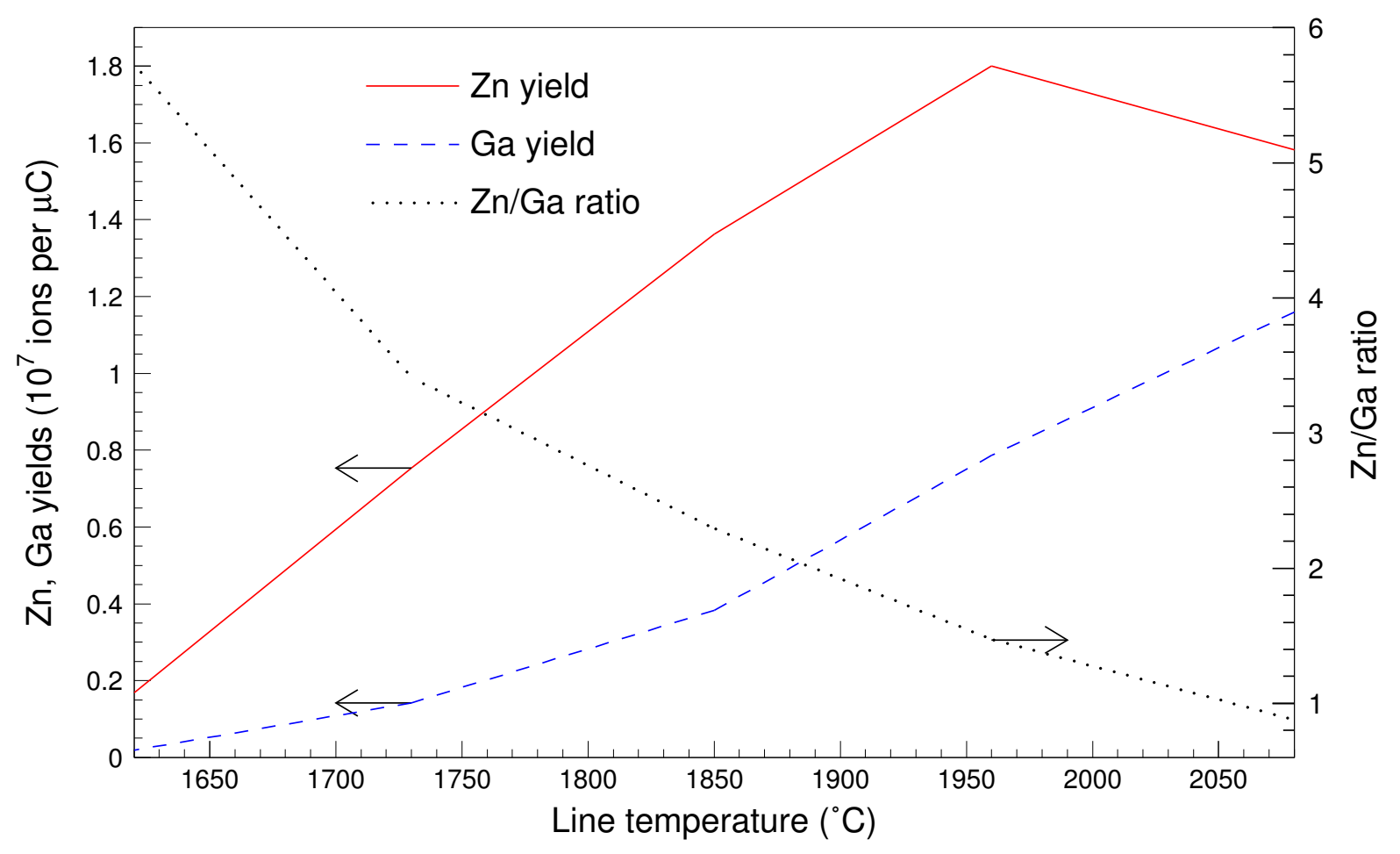

Figure 1: Dependence of the zinc and gallium yields on the line temperature

Although the RILIS does not suppress all background, it can allow identifying it by comparing "laser on" and "laser off" spectra. For the latter, one or all the ionizing laser beams are blocked or detuned in frequency. The difference between "laser on" and "laser off" spectra shows then the net signal due to the laser-ionized elements.

\subsection{Neutron converter}

When inducing fission of ${ }^{238} \mathrm{U}$ by $\mathrm{GeV}$ protons, there will be a strong contribution of spallation-fission, i.e. part of the target nuclei will first evaporate a significant number of mainly neutrons and then fission in a later stage. This leads to a shift of the fission yields towards less neutron-rich or even to neutron-deficient species [12]. In the mass region $A \approx 80$ this effect leads to significant background from neutron-deficient rubidium isotopes [5]. To avoid such background it is preferable to use low-energy fission, which only produces neutron-rich species. This can be done by sending the $\mathrm{GeV}$ protons not directly onto the $\mathrm{UC}_{x}$ target, but onto a heavy metal rod (e.g. tungsten) mounted close to the target. The spallation neutrons produced in the latter have mainly energies in the $\mathrm{MeV}$ region [13]. Part of them are emitted in radial direction and can thus interact with the $\mathrm{UC}_{x}$ target mounted parallel to the metal rod. The latter thus acts as an efficient "converter" of $\mathrm{GeV}$ protons into $\mathrm{MeV}$ neutrons and is therefore often called "neutron converter". The use of the neutron converter is not sufficient for a unique selection of an isotope, but helps as a supplementary measure to enhance the selection power of the two previously mentioned methods.

Target UC2.280 was equipped with a $125 \mathrm{~mm}$ long W converter of $12.7 \mathrm{~mm}$ diameter, mounted parallel to the target at a distance of $23.5 \mathrm{~mm}$ (center to center). Longitudinally the middle of the converter and of the target coincided. 


\subsection{Macro-Time structure}

Due to the different diffusion and effusion times, different elements are released more or less quickly. In the present case zinc shows a clearly quicker release than the unwanted gallium, see table 1 and figure 2. Thus Ga background can be suppressed by an additional factor two to four by only sending the radioactive ion beam to the experiment during the first 0.2 to $0.6 \mathrm{~s}$ after proton impact.

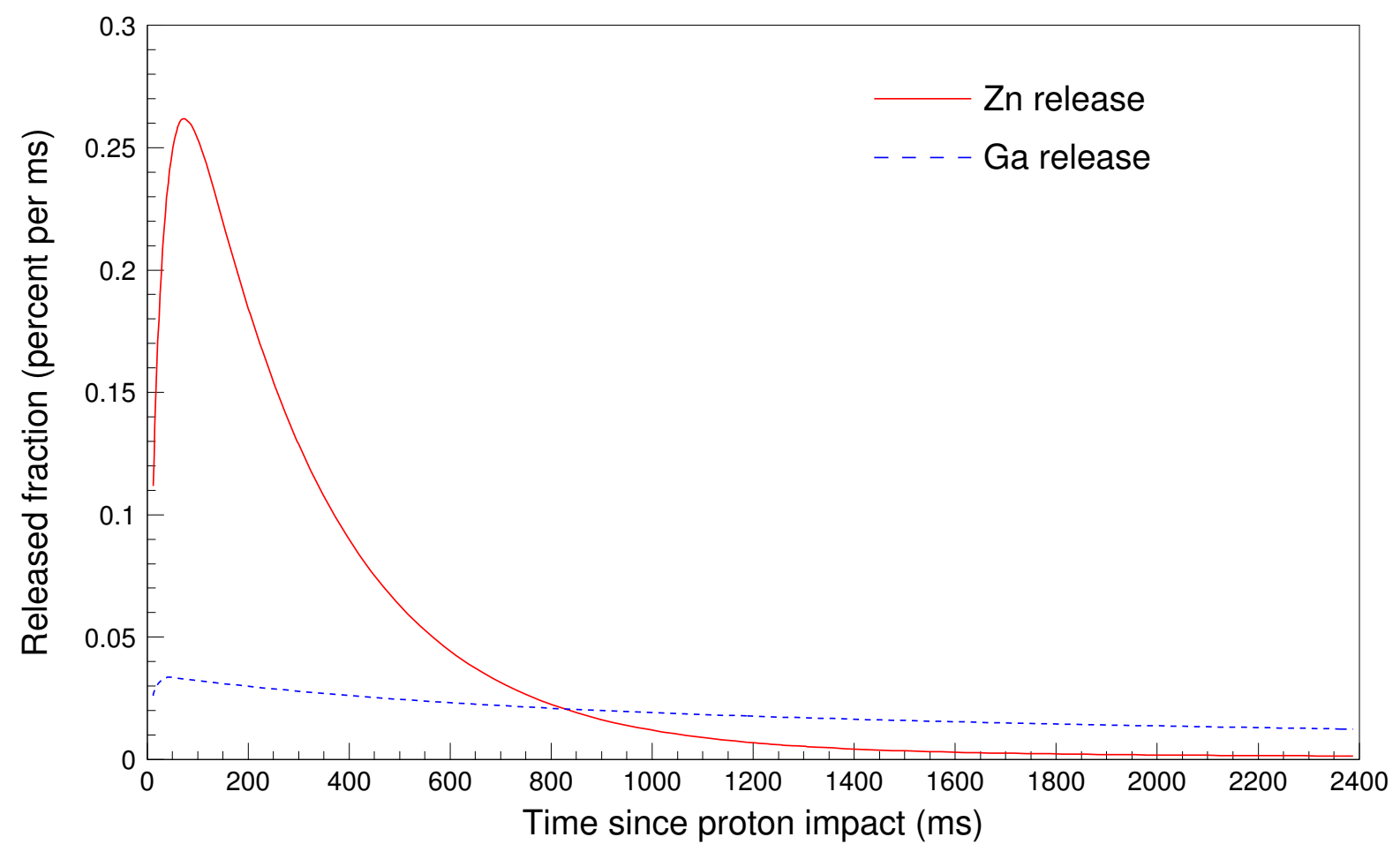

Figure 2: Typical release time profile of zinc and gallium.

\section{$3 \quad$ Yields and Release}

The yields (i.e. radioactive ion beam intensity normalized to $1 \mu \mathrm{A}$ intensity of incident proton beam), have been determined either on- or off-line by Faraday cup measurements or beta- or gamma spectrometry respectively. To determine the release speed only one proton pulse every $14.4 \mathrm{~s}, 16.8 \mathrm{~s}$ or $19.2 \mathrm{~s}$ was sent onto the target. The amount of released radioactive ions was then measured as function of time since the last proton pulse. Figure 2 shows representative release time profiles of stable $\mathrm{Zn}$ and Ga and table 1 gives the release parameters obtained by fitting the measured curves with the empiric four-parameter formula [14]:

$$
P(t)=N\left(1-e^{-t \ln (2) / t_{r}}\right)\left[\alpha e^{-t \ln (2) / t_{f}}+(1-\alpha) e^{-t \ln (2) / t_{s}}\right]
$$

As the release parameters and yields evolve during an on-line run, the time is included in table 1 for which the target has been exposed to the proton beam. Typically the release gets slower with an ageing target. The ${ }^{74,76} \mathrm{Zn}$ yields of target UC2.259 dropped by a factor two to three during the 7 days of operation. The target deterioration is mainly due to the thermal spikes and mechanical stress during the impact of the intense proton pulses.

In all cases where both isomers were observed for the odd mass zinc isotopes, the yield of the high spin isomer was higher than of the low spin isomer. The yield values 


\begin{tabular}{|c|c|c|c|c|c|c|c|c|c|}
\hline $\begin{array}{c}\text { Ele- } \\
\text { ment }\end{array}$ & $\begin{array}{c}\text { Target } \\
\text { Number }\end{array}$ & $\begin{array}{c}\text { On-line } \\
\text { run time } \\
(\mathrm{h})\end{array}$ & $\begin{array}{c}\text { Target } \\
\text { temp. } \\
\left({ }^{\circ} \mathrm{C}\right)\end{array}$ & $\begin{array}{c}\text { Line } \\
\text { temp. } \\
\left({ }^{\circ} \mathrm{C}\right)\end{array}$ & $\begin{array}{c}\text { proton } \\
\text { beam } \\
(1.4 \mathrm{GeV})\end{array}$ & $t_{r}$ & $t_{f}$ & $t_{s}$ & $\alpha$ \\
$(\mathrm{ms})$ & $(\mathrm{ms})$ & $(\mathrm{ms})$ & \\
\hline $\mathrm{Zn}$ & $\mathrm{UC} 2.259$ & 1 & 2000 & 2030 & onto target & 26 & 125 & 2000 & 0.991 \\
$\mathrm{Zn}$ & $\mathrm{UC} 2.259$ & 120 & 1900 & 2120 & onto target & 23 & 190 & 1500 & 0.989 \\
$\mathrm{Ga}$ & $\mathrm{UC} 2.259$ & 120 & 1900 & 2120 & onto target & 6 & 400 & 3060 & 0.40 \\
$\mathrm{Zn}$ & $\mathrm{UC} 2.280$ & 1 & 2060 & 2040 & onto conv. & 30 & 200 & 1600 & 0.98 \\
\hline
\end{tabular}

Table 1: Target and ion source characteristics and measured release parameters.

for ${ }^{62} \mathrm{Zn}$ and ${ }^{65} \mathrm{Zn}$ suffer from a larger uncertainty, due to a varying transmission to the collection chamber.

With the known release function, one can calculate for each isotope what fraction will decay inside the target before it is released. Correcting for this loss and assuming an ionization efficiency of $5 \%$, one can calculate back to the "in-target production rates" of the $\mathrm{Zn}$ isotopes. Comparing the latter with the production rates calculated from crosssections measured in inverse kinematics at $1 \mathrm{GeV} /$ nucleon at GSI-FRS [12] indicates that the on-line ionization efficiency was probably slightly higher (7-8\%). For gallium a good agreement between our in-target production rates and the GSI cross-sections is obtained by assuming $1.5 \%$ ionization efficiency, see figure 3 .

The yields measured with the proton beam sent onto the target UC2.280 were a factor two to three lower compared to those shown from target UC2.259. This might be due to a lower ionization efficiency. Both targets had a thickness of $52 \mathrm{~g} / \mathrm{cm}^{2}{ }^{238} \mathrm{U}$.

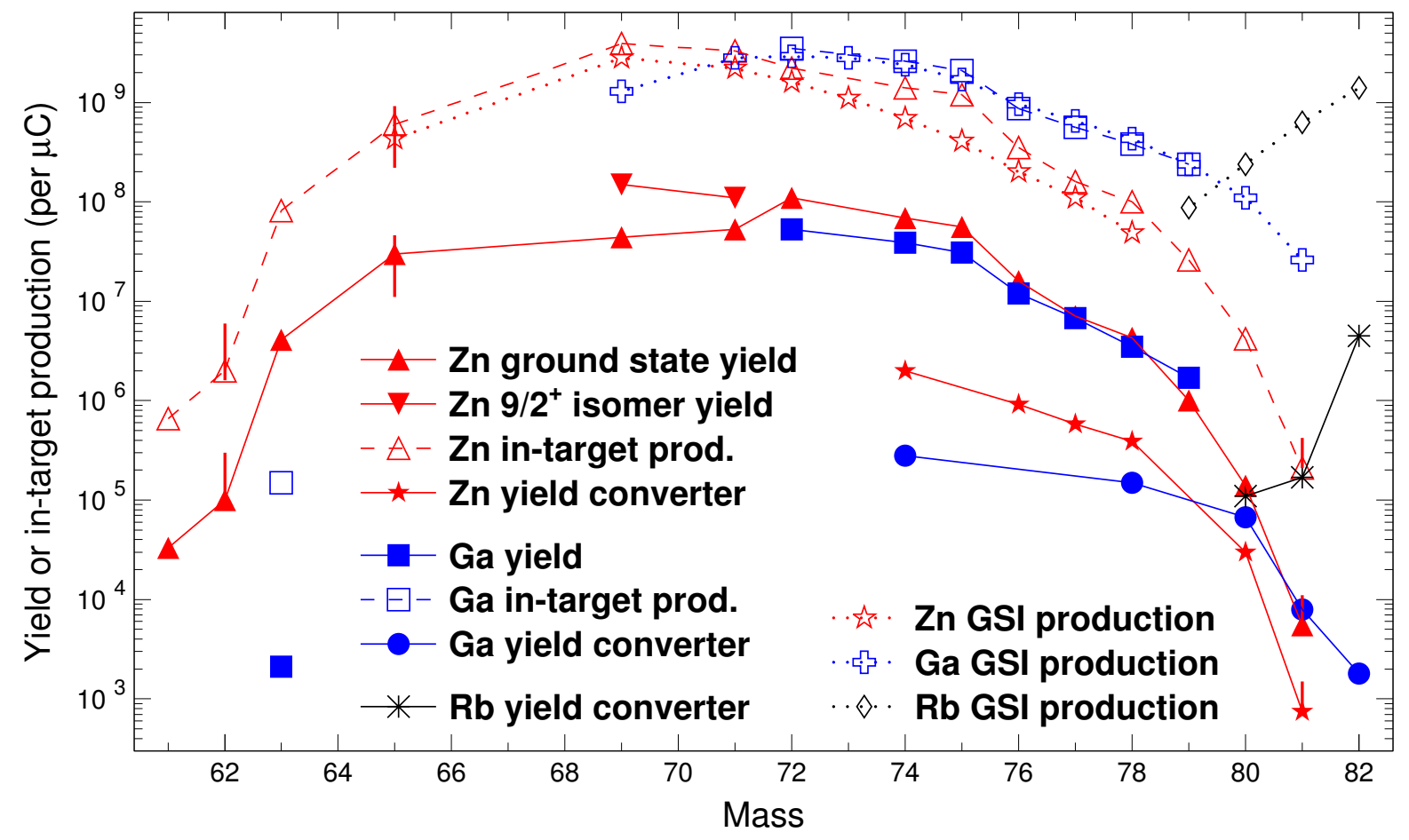

Figure 3: Yields (filled symbols) and estimated in-target production rates (open symbols) of $\mathrm{Zn}, \mathrm{Ga}$ and $\mathrm{Rb}$ isotopes. The "converter yields" were measured with the proton beam sent onto the converter of the target UC2.280, all other yields with the proton beam sent directly onto the target UC2.259. The lines are drawn to guide the eye. 
Beams of ${ }^{74,76,78} \mathrm{Zn}$ have been post-accelerated with REX to measure the $B(\mathrm{E} 2)$ values by Coulomb excitation. At masses 74 and 76 the beam purity was sufficient, even when directing the proton beam directly onto the target. At mass 78 however, strong Rb and Ga background was present. When directing the proton beam onto the neutron converter the $\mathrm{Rb}$ background practically vanished. Moreover the $\mathrm{Zn}$ to Ga ratio was improved. Fig. 4 shows as $\mathrm{E}_{\text {total }}-\Delta \mathrm{E}$ plot the composition of the beam post-accelerated to $2.86 \mathrm{MeV}$ per nucleon and measured with an ionization chamber. The figure shows the beam composition when directing the proton beam onto the target or converter respectively.
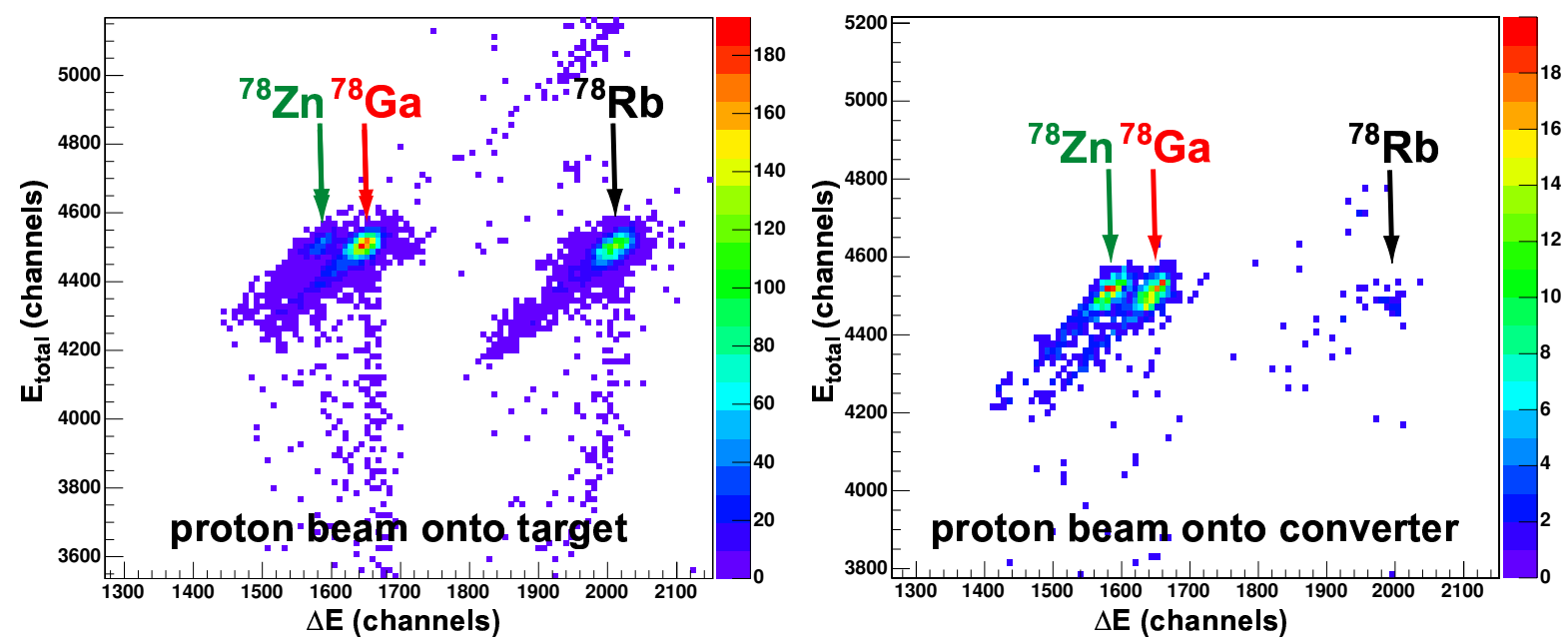

Figure 4: Beam composition of post-accelerated mass 78 beams with the proton beam sent onto the target (left) and the converter (right) respectively.

\section{$5 \quad$ Decay spectroscopy}

While the yield measurement of longer-lived, more abundantly produced isotopes can be easily performed by the methods mentioned before, the detection of the most exotic isotopes requires a more elaborate set-up. In the case of zinc this becomes necessary for ${ }^{81} \mathrm{Zn}$. Figure 5 shows gamma spectra taken on mass 81 . Comparing the "laser on" (96 pulses of $3.5 \mu \mathrm{C}$ protons each, $403 \mathrm{~s}$ measurement) and "laser off" spectra (61 pulses of $3.5 \mu \mathrm{C}$ protons each, $255 \mathrm{~s}$ measurement) allows to clearly identify the $351 \mathrm{keV}$ and $452 \mathrm{keV}$ lines belonging to the decay of ${ }^{81} \mathrm{Zn}$. This confirms the assignment from a recent measurement of the ${ }^{81} \mathrm{Zn}$ decay performed at the PARRNe separator at IPN Orsay [15].

The ${ }^{81} \mathrm{Zn}$ yield shown in Fig. 3 is actually a lower limit as it was deduced assuming $100 \%$ absolute branching ratio for the $351 \mathrm{keV}$ gamma ray. Probably this branching ratio is indeed rather high (>50\%), else a more pronounced difference in ${ }^{81} \mathrm{Ga}$ intensity in the "laser on" and "laser off" spectra should be observed. One also observes a significant ${ }^{80} \mathrm{Ga}$ activity in the ${ }^{81} \mathrm{Zn}$ decay spectrum that suggests a $P_{n}$ value $>10 \%$ for ${ }^{81} \mathrm{Zn}$, which would be higher than the previously measured $P_{n}=7.5(30) \%[9]$.

\section{Conclusion and outlook}

It has been shown that clean and intense beams of neutron-rich $\mathrm{Zn}$ isotopes can be produced at ISOLDE by combining resonant laser ionization with a neutron converter. The beam purity was sufficient to easily perform decay spectroscopy up to ${ }^{81} \mathrm{Zn}$ and Coulomb 


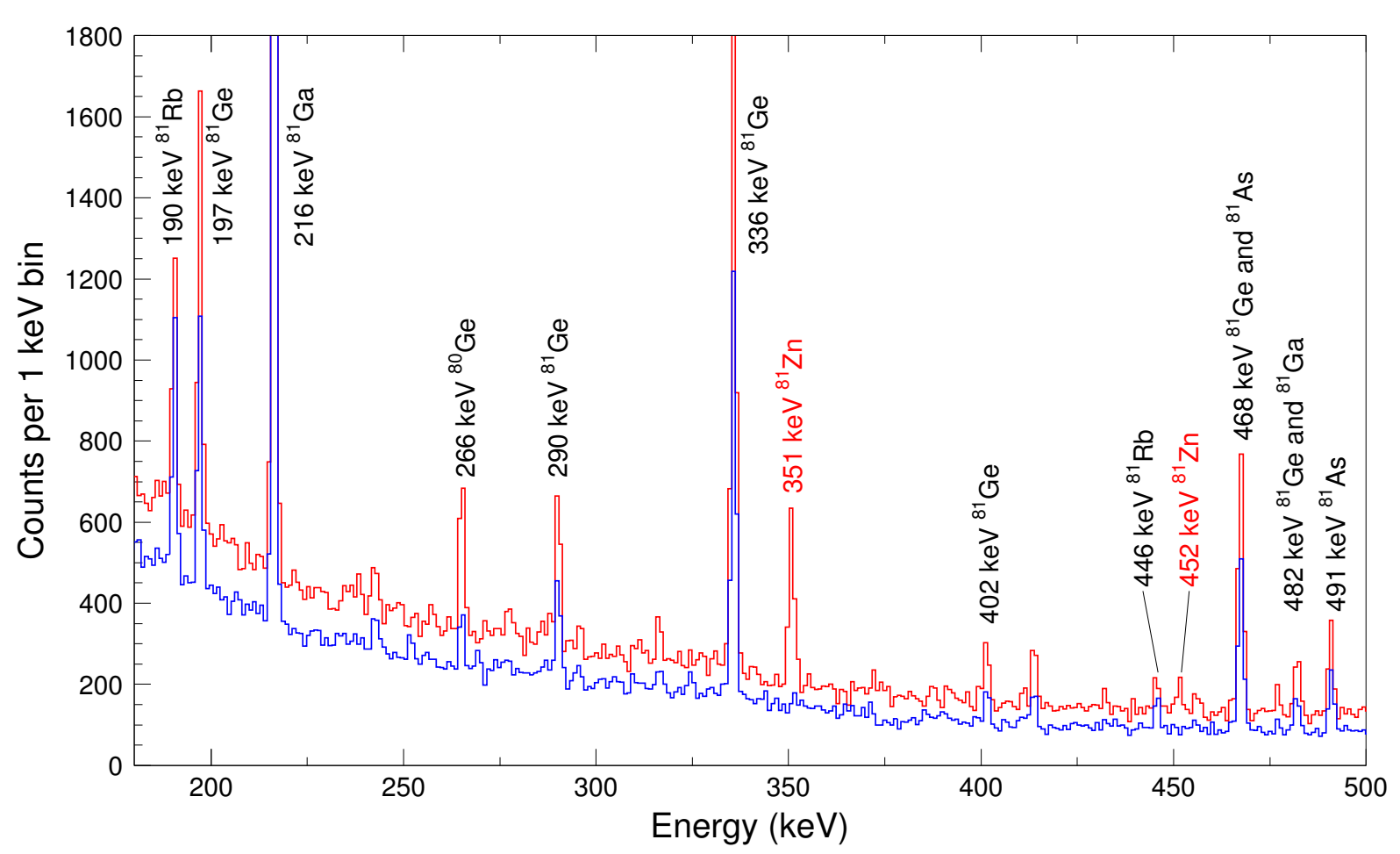

Figure 5: Part of the gamma ray spectrum measured on mass 81 in the "laser on" (upper red histogram) and "laser off" (lower blue histogram) mode respectively.

excitation measurements up to ${ }^{78} \mathrm{Zn}$. To proceed to still heavier $\mathrm{Zn}$ isotopes, the beam purity has to be further increased without compromising the $\mathrm{Zn}$ beam intensity. A possible way is the combination of the present measures with a chemically selective transfer line. In preparatory off-line thermochromatography experiments by the TARGISOL collaboration it has been found that a transfer line made from quartz or different types of glass could help to retain $\mathrm{Ga}$ and $\mathrm{Rb}$ without delaying $\mathrm{Zn}$ too much [16]. A test of such a prototype target at ISOLDE combining a chemically selective transfer line with resonant laser ionization is planned soon. It should make possible the future use of ${ }^{80} \mathrm{Zn}$ for Coulomb excitation experiments and of ${ }^{82} \mathrm{Zn}$ or even ${ }^{83} \mathrm{Zn}$ for $\beta \gamma$ decay spectroscopy.

\section{Acknowledgments}

Supported by the EU-RTD project TARGISOL (HPRI-CT-2001-50033). We thank the IS386 collaboration for "squatting" their $\gamma$-spectroscopy set-up for the measurement of the ${ }^{77-81} \mathrm{Zn}$ yields and the IS412 collaboration for providing figure 4 .

\section{References}

[1] H. Ravn, S. Sundell, L. Westgaard, and E. Roeckl, J. Inorg. Nucl. Chem., 37, 383-393 (1975).

[2] F. Wenander, J. Lettry, and M. Lindroos, Nucl. Instr. Meth. B, 204, 261-266 (2003).

[3] M. Oinonen, et al., Hyp. Int., 127, 431-436 (2000).

[4] A. Jokinen, et al., Nucl. Instr. Meth. B, 126, 95-99 (1997).

[5] U. Köster, Eur. Phys. J. A, 15, 255-263 (2002).

[6] U.C. Bergmann, et al., Nucl. Instr. Meth. B, 204, 220-224 (2003).

[7] U. Köster, et al., Nucl. Instr. Meth. B, 204, 347-352 (2003).

[8] I. Dillmann, et al., Eur. Phys. J. A, 13, 281-284 (2002). 
[9] K.L. Kratz, et al., Z. Phys. A, 340, 419-420 (1991).

[10] U. Köster, V. Fedoseyev, and V. Mishin, Spectrochimica Acta B, 58, 1047-1068 (2003).

[11] J. Lettry, et al., Rev. Sci. Instr., 69, 761-763 (1998).

[12] M. Bernas, et al., Nucl. Phys. A, 725, 213-253 (2003).

[13] R. Catherall, J. Lettry, S. Gilardoni, U. Köster, and the ISOLDE Collaboration, Nucl. Instr. Meth. B, 204, 235-239 (2003).

[14] J. Lettry, et al., Nucl. Instr. Meth. B, 126, 130-134 (1997).

[15] D. Verney, et al., Brazilian J. Phys., 34, 979-982 (2004).

[16] C. Jost, Thermochromatographische Experimente zur Ionenquellenentwicklung, Diplomarbeit, Johannes-Gutenberg-Universität, Mainz (2005). 